\title{
Hybrid spatial uncertainty analysis for the estimation of imprecise failure probabilities in Laser Sintered PA-12 parts
}

\author{
Matthias Faes ${ }^{\mathrm{a}, *}$, Sabyasachi Ghosh Dastidar ${ }^{\mathrm{a}}$, David Moens ${ }^{\mathrm{a}}$ \\ ${ }^{a} K U$ Leuven - Department of Mechanical Engineering, Jan De Nayerlaan 5, 2860 \\ St.-Katelijne-Waver, Belgium
}

\begin{abstract}
This paper presents the application of non-determinstic modelling techniques for the quantification of imprecise failure probabilities in PA-12 parts produced via Laser Sintering (LS). Specifically, a modelling approach consisting of a combination of interval and random fields is applied, and the effect hereof on the resulting polymorphic uncertainty in the mechanical performance of the LS parts is studied. A link with the underlying process physics is given, relating the spatial porosity distributions that are inherently present in LS PA12 parts to a spatial distribution in the apparent Young's modulus. Furthermore, uncertainty on the final dimensions of other parts that are constructed in the same build envelope as the designed part is linked to uncertainty in the cross-sectional area of the designed part.

The effect of these sources of uncertainty is studied in three case studies, illustrating the need for a well-thought production process planning given the influence on the resulting mechanical performance of the designed structure. Especially the effect of epistemic uncertainty on the design process is shown to be significant.
\end{abstract}

Keywords: Uncertainty quantification, Random Fields, Interval Fields, hybrid uncertainty, Laser Sintering, Polymorphic Uncertainty

\footnotetext{
${ }^{*}$ Corresponding author

Email address: matthias.faes@kuleuven.be (Matthias Faes)

URL: https://iiw.kuleuven.be/onderzoek/op/research/r2d (Matthias Faes)
} 


\section{Introduction}

Additive manufacturing (AM) enables designers of end-use components to effectively produce the highly optimized geometries that result from advanced design optimization methods without being constrained by conventional designfor-manufacture ideologies. In the context of producing plastic end-use parts, especially Laser Sintering (LS) is deemed to be one of the most robust manufacturing processes [1]. LS builds up complex parts in a layer-by-layer approach by selectively irradiating a preheated powder bed of polymeric particles by means of a high power laser, selectively sintering the material [2].

However, inter- (between nominally identical parts) and intra- (within one realization of a part) variability and uncertainty exist at both the micro, meso and macro scale. In this context, variability is used to refer to sources that are inherently random (aleatory), whereas uncertainty is used to refer to a lack of knowledge on the underlying effects (epistemic). Note that these phenomena are not mutually exclusive [3. Even for PA-12, the most researched LS material, a considerable amount of non-determinism in the mechanical and dimensional properties was found in previous work (see e.g., [4, [5, 6]). At the micro-scale, for example, the mechanical material behavior is mainly affected by the local amount of pores and their topology, caused by insufficient sintering and limited powder flow due to short processing times 6, 7. On the meso-scale, non-determinism in part properties such as the local stiffness and dimensional accuracy is caused by changes in inter-layer time (i.e. the time between the scanning of a certain point of the layer and the recoating operation) between subsequent layers of the part [4, 6]. Finally, on the macro-scale of the part, it has been shown that also the location in the build envelope influence the resulting mechanical properties due to inhomogeneities in the pre-heating temperature and the post-building cool-down of the components 8, 4. Many other complicated interactions between process parameters, stock material properties and process planning influence these properties. For an elaborate review, the 

production process and typically produced parts. Section 2 first introduces the most important concepts concerning variability and uncertainty, provides a rigorous mathematical explanation of the framework of random fields and interval fields and presents the propagation of the hybrid uncertain model. Also a posmodel is sketched. In section 3, it is illustrated how such a hybrid model can be constructed from a priori obtained measurement data and which modelling assumptions should be made in such a context. Also the results of propagating such hybrid uncertainty through the numerical model are discussed. Finally, applicability of this hybrid uncertainty model. 


\section{Non-deterministic modelling of spatial non-determinism}

In the context of including non-determinism into numerical design models, two complementary philosophies exist following either probabilistic or possideterministic parameters using (joint-) probability density functions. The latter approach considers only the crisp bounds between which the possible values of the corresponding parameter lie for analysis. An elaborate amount of literature has been dedicated to the comparison of both classes of methods [9, 3] showing achieved when the parameters under consideration call for a different modelling approach. As such, a hybrid uncertain model is obtained. However in this case, the analyst has to keep track of the sources of the non-determinism in the model responses as to differentiate them accordingly [10]. solution of sets of partial differential equations (PDE). The approximative solution of these PDE's is usually provided by means of a numerical model $\mathcal{M}(\boldsymbol{x})$, parametrized by a parameter vector $\boldsymbol{x}(\mathbf{r}) \in \mathcal{X} \subset \mathbb{R}^{k}$ with $\mathcal{X}$ the set of physically admissible parameters and $k \in \mathbb{N}$. For example, $\boldsymbol{x}(\mathbf{r})$ may contain inertial function of a spatial coordinate $\mathbf{r} \in \Omega \subset \mathbb{R}^{d}$ over the model domain $\Omega$ with dimension $d \in \mathbb{N}$. In case $\mathcal{M}(\boldsymbol{x})$ is constructed following a finite element approach, $\Omega$ is discretised by means of a set of finite elements, yielding $d$ structural degrees of freedom (DOF).

The model $\mathcal{M}(\boldsymbol{x})$ provides a vector of model responses $\boldsymbol{y}(\mathbf{r}) \in \mathcal{Y} \subset \mathbb{R}^{l}$, with $\mathcal{Y}$ the set of admissible model responses and $l \in \mathbb{N}$, through a set of function operators $m_{i}, i=1, \ldots, l$, which are defined as:

$$
\mathcal{M}(\boldsymbol{x}): y_{i}(\mathbf{r})=m_{i}(\boldsymbol{x}(\mathbf{r})) \quad i=1, \ldots, l
$$

80

with $m_{i}: \mathbb{R}^{k} \mapsto \mathbb{R}$

Note that the dependence of $\boldsymbol{y}$ on $\mathbf{r}$ is only valid when nodal or elemental 
responses are considered. This is for example not the case when $\boldsymbol{y}$ consists of eigenfrequencies.

\subsection{Random field case}

The probabilistic concept is highly suited for characterizing an inherent variability because the available information on the range of possible values and likelihood of each value within this range can perfectly be described using a probability density function (PDF). For this to be feasible, the correlation between all non-deterministic quantities needs to be quantified with sufficient accuracy

[3. When such data are not available, the analysis automatically becomes subjective. In that case, interval methods provide the analyst with more objective extreme bounds on the uncertainty, albeit that the information on the likelihood of each parameter value is omitted. The interval methods are discussed in detail in Section 2.2.

In a probabilistic context, model parameters $\boldsymbol{x}(\mathbf{r})$ that are subjected to spatial variability are usually modelled as a random field $x(\mathbf{r}, \theta)$. Such a random field $x(\mathbf{r}, \theta)$ describes a set of correlated random variables $x(\theta)$ for each location $\mathbf{r} \in \Omega$ in the continuous model domain $\Omega \subset \mathbb{R}^{d}$ with dimension $d \in \mathbb{N}$. Each such a random variable $x(\theta)$ provides a mapping $x:(\Theta, \sigma, P) \mapsto \mathbb{R}$ with $\theta \in \Theta$ a coordinate in sample space $\Theta$ and $\sigma$ the sigma-algebra. For a given event $\theta_{i}, x\left(\mathbf{r}, \theta_{i}\right)$ is a realisation of the random field. A random field is considered Gaussian if the distribution of $\left(x\left(\mathbf{r}_{1}, \theta\right), x\left(\mathbf{r}_{2}, \theta\right), \ldots, x\left(\mathbf{r}_{n}, \theta\right)\right)$ is jointly Gaussian $\forall \mathbf{r} \in \Omega$. In this case, $x(\mathbf{r}, \theta)$ is completely described by its mean function $\mu_{x}(\boldsymbol{r}): \Omega \mapsto \mathbb{R}$ and its auto-covariance function $\boldsymbol{\Gamma}_{x}\left(\mathbf{r}, \mathbf{r}^{\prime}\right): \Omega \times \Omega \mapsto \mathbb{R}$.

105 The application of random fields in the context of non-deterministic numerical modelling requires a discretization over $\Omega$. Usually, this is obtained following a Karhunen-Loève (KL) series expansion [11, 12, which is based on the representation of $x(\mathbf{r}, \theta)$ as a truncated series expansion. Considering a continuous, bounded, symmetric and positive definite auto-covariance function $\boldsymbol{\Gamma}_{x}\left(\mathbf{r}, \mathbf{r}^{\prime}\right): \Omega \times \Omega \mapsto \mathbb{R}$ of the random field $x(\mathbf{r}, \theta)$, the application of Mercer's 
theorem yields following spectral decomposition:

$$
\boldsymbol{\Gamma}_{x}\left(\mathbf{r}, \mathbf{r}^{\prime}\right)=\sum_{i=1}^{\infty} \lambda_{i} \boldsymbol{\psi}_{i}(\mathbf{r}) \boldsymbol{\psi}_{i}\left(\mathbf{r}^{\prime}\right)
$$

where $\lambda_{i} \in[0, \infty)$ and $\boldsymbol{\psi}_{i}: \Omega \mapsto \mathbb{R}$ are respectively the eigenvalues and eigenfunctions of $\boldsymbol{\Gamma}_{x}\left(\mathbf{r}, \mathbf{r}^{\prime}\right)$, obtained by solving the homogeneous Fredholm integral equation of the second kind:

$$
\int_{\Omega} \boldsymbol{\Gamma}_{x}\left(\mathbf{r}, \mathbf{r}^{\prime}\right) \boldsymbol{\psi}_{i}\left(\mathbf{r}^{\prime}\right)=\lambda_{i} \boldsymbol{\psi}_{i}(\mathbf{r})
$$

Since $\boldsymbol{\Gamma}_{x}\left(\mathbf{r}, \mathbf{r}^{\prime}\right)$ is bounded, symmetric and positive definite, these eigenvalues $\lambda_{i}$ are non-negative and the eigenfunctions $\boldsymbol{\psi}_{i}(\mathbf{r})$ satisfy the orthogonality condition as $\int_{\Omega} \boldsymbol{\psi}_{i}(\mathbf{r}) \boldsymbol{\psi}_{j}(\mathbf{r}) d \mathbf{r}=\delta_{i j}$, with $\delta_{i j}$ the Kronecker delta.

In this case, the series expansion in eq. (2) is shown to be convergent [11]. As such, the random field can be expressed as a series expansion:

$$
H(\mathbf{r}, \theta)=\mu_{x}(\mathbf{r})+\sum_{i=1}^{\infty} \sqrt{\lambda_{i}} \boldsymbol{\psi}_{i}(\mathbf{r}) \xi_{i}(\theta)
$$

with $\xi_{i}(\theta), i=1, \ldots, \infty$ standard uncorrelated random variables, which are determined following:

$$
\xi_{i}(\theta)=\frac{1}{\sqrt{\lambda_{i}}} \int_{\Omega}\left[x(\mathbf{r}, \theta)-\mu_{x}(\mathbf{r})\right] \boldsymbol{\psi}_{i}(\mathbf{r}) d \mathbf{r}
$$

which are independent standard normal in the case of a Gaussian random field. In case the field is non-Gaussian, the joint distribution of $\xi_{i}(\theta)$ is very hard to obtain. Therefore, non-Gaussian random fields are typically processed as functions of Gaussian random fields (see e.g., [13, 14] for a treatment of non-Gaussian random fields). In this paper, only Gaussian random fields are considered, but the presented ideas are easily extended to non-Gaussian random fields.

In order to limit the computational cost, the series expansion in eq. (4) is usually truncated by retaining only the $m \in \mathbb{N}$ largest eigenvalues and corresponding eigenfunctions of $\boldsymbol{\Gamma}_{x}\left(\mathbf{r}_{i}, \mathbf{r}_{j}\right)$ [15], which yields an optimal series expansion with respect to the global mean squared error [16]. Formally, this is 
expressed as:

$$
\tilde{\boldsymbol{H}}(\mathbf{r}, \theta)=\mu_{x}(\mathbf{r})+\sum_{i=1}^{m} \sqrt{\lambda_{i}} \boldsymbol{\psi}_{i}(\mathbf{r}) \xi_{i}(\theta)
$$

Finally, based on this discretized random field, realizations $\tilde{\boldsymbol{H}}\left(\mathbf{r}, \theta_{i}\right), i=$ $1, \ldots, N$ are generated over $\Omega$ by drawing $N \in \mathbb{N}$ samples from the independent standard normal random variables $\xi_{i}(\theta), i=1, \ldots, m$ following a Monte Carlo sampling approach. Then, each of these realizations are propagated through the numerical model $\mathcal{M}$ :

$$
\boldsymbol{y}\left(\mathbf{r}, \theta_{i}\right)=\mathcal{M}\left(\tilde{\boldsymbol{H}}\left(\mathbf{r}, \theta_{i}\right)\right) \quad i=1, \ldots, N
$$

From these propagated realisations, a probabilistic description $\boldsymbol{y}(\mathbf{r}, \theta)$ of the model's responses $\boldsymbol{y}(\mathbf{r})$ is inferred. Generally, $N$ should be sufficiently large to allow for an accurate estimation of the joint probability structure of $\boldsymbol{y}(\mathbf{r}, \theta)$.

\subsection{Interval field case}

Interval fields are appropriate for characterizing the non-determinism in the model parameters when they stem from a pure lack of knowledge, rather than from a variability. In this case, the application of probability density functions results in unrealistic model behavior since it does not represent a physical variation [17. Furthermore, the spatial correlation structure of the random field is often very cumbersome to obtain, which might hinder objective analysis under insufficient knowledge. Interval fields provide the analyst with less information, at the advantage that also less information is needed to obtain an objective quantification of the response uncertainty.

Opposed to random fields, where the description of the spatial variability is inherently embedded within the probabilistic framework, the definition and discretization of spatial uncertainty in an interval context is more challenging since intervals are by construction independent. Therefore, interval field [18, 19] approaches were recently introduced to model parameters $\boldsymbol{x}$ that are subjected to spatial uncertainty. A model parameter $x$ having interval uncertainty is denoted $x^{I}$. Interval parameters are either represented using the bounds of 
the interval $x^{I}=[\underline{x} ; \bar{x}]$ or the centre point $\hat{x}=\frac{\underline{x}+\bar{x}}{2}$ and the interval radius $r_{x}=\frac{\bar{x}-\underline{x}}{2}$. The set of real interval-valued scalars is denoted $\mathbb{R}$.

Following the method of Moens et al. [18, the description of an interval field is based on the superposition of $n_{b} \in \mathbb{N}$ base functions $\boldsymbol{\psi}_{i}(\mathbf{r}): \Omega \mapsto \mathbb{R}$, which represent a set of spatial uncertainty patterns, scaled by independent interval scalars $\alpha_{i}^{I} \in \mathbb{R}$, which represent magnitude of the spatially uncertain model parameters. Following the series expansion over these base functions, a mapping from the model domain $\Omega$ to a reduced $n_{b}$ dimensional input space is provided. Formally, an interval field for the description of spatial uncertainty in the input parameters $\boldsymbol{H}^{\boldsymbol{I}}(\mathbf{r})$ is expressed in the explicit form as:

$$
\boldsymbol{H}^{\boldsymbol{I}}(\mathbf{r})=\sum_{i=1}^{n_{b}} \alpha_{i}^{I} \boldsymbol{\psi}_{i}(\mathbf{r})
$$

When $\Omega$ is discretized into $k$ finite elements, the discretized base functions $\boldsymbol{\psi}_{i}(\mathbf{r}) \in \mathbb{R}^{k}$ interpolate the interval scalars $\alpha_{i}^{I}$ to intervals for each element in $\Omega$. As such, it is also clear that, when $n_{b}<k$, a reduction of the input space dimension is obtained.

For practical application, the definition of the base functions $\boldsymbol{\psi}_{i}(\mathbf{r})$ in 8 under scarce information, proves to be a non-trivial task as these base functions should be able to translate expert knowledge of the analyst on the spatial nature of the uncertainty to a mathematical formulation in an intuitive way, while delivering a realistic representation of this uncertainty. This expert knowledge can stem e.g., from engineering judgement, production process knowledge and/or physical simulation models of the production process. In this context, the Inverse Distance Weighting (IDW) interpolation method was recently applied by [20, 21]. Following an IDW approach, the base functions $\boldsymbol{\psi}_{i}(\mathbf{r})$ are constructed according to:

$$
\boldsymbol{\psi}_{i}(\mathbf{r})=\frac{w_{i}(\mathbf{r})}{\sum_{j=1}^{n_{b}} w_{j}(\mathbf{r})}
$$

with $w_{i}(\mathbf{r}) \in \Omega$ and $i=1, \ldots, n_{b}$ :

$$
w_{i}(\mathbf{r})=\frac{1}{\left[d\left(\mathbf{r}_{i}, \mathbf{r}\right)\right]^{p}}
$$


with $p \in \mathbb{R}^{+}$. Finally, the interval field is then explicitly defined as:

$$
\boldsymbol{H}^{\boldsymbol{I}}(\mathbf{r})=\sum_{i=1}^{n_{b}} \frac{w_{i}(\mathbf{r}) \alpha_{i}^{I}}{\sum_{j=1}^{n_{b}} w_{j}(\mathbf{r})}
$$

The solution of a model $\mathcal{M}$ containing interval field uncertain parameters $\boldsymbol{H}^{\boldsymbol{I}}(\mathbf{r})$ is aimed at finding those realizations of the interval field that yield extrema in the set of model responses $\tilde{\boldsymbol{y}}(\mathbf{r})$, obtained by propagating the interval field:

$$
\tilde{\boldsymbol{y}}(\mathbf{r})=\left\{\boldsymbol{y}_{i}(\mathbf{r}) \mid \boldsymbol{y}_{i}(\mathbf{r})=\mathcal{M}\left(\boldsymbol{H}_{F}^{(i)}(\mathbf{r})\right) ; \boldsymbol{H}_{F}^{(i)}(\mathbf{r}) \in \boldsymbol{H}_{F}^{I}(\mathbf{r}) ; i=1, \ldots, q\right\}
$$

with $q \in \mathbb{N}$ the number of propagated realizations of the interval field [22]. $\tilde{\boldsymbol{y}}(\mathbf{r})$ is obtained either directly via affine arithmetical methods [23, 24, or indirectly following a global optimization approach [25]. Alternatively, also advanced sampling approaches can be applied [26]. In the specific case that $\mathcal{M}$ is monotonous, the transformation method [27, 28] yields the best convex approximation of the solution set $\tilde{\boldsymbol{y}}(\mathbf{r})$.

\subsection{Hybrid probability of failure analysis}

In order to estimate the reliability of the component or structure in a probabilistic context, usually the probability of failure $P_{f}=P\left(\mathcal{M}(\boldsymbol{x}(\mathbf{r}, \theta)) \in \mathcal{Y}_{\mathcal{F}}\right)$ is computed. Herein, $\mathcal{Y}_{\mathcal{F}}=\{\boldsymbol{y}(\mathbf{r}, \theta) \in \mathcal{Y} \mid g(\boldsymbol{y}(\mathbf{r}, \theta)) \leq 0\}$ is the failure domain of the model, given the random field uncertain model parameters. The special case $\mathcal{Y}_{\mathcal{F}}^{\prime}=\{\boldsymbol{y}(\mathbf{r}, \theta) \in \mathcal{Y} \mid g(\boldsymbol{y}(\mathbf{r}, \theta))=0\}$ is called the limit-state surface, which is completely independent of $\mathcal{Y}$, but governed by structure-specific safety demands and the underlying numerical model.

In a purely probabilistic context, the probability of failure $P_{f} \in[0,1]$ is determined as:

$$
P_{f}=\int_{\mathcal{Y}_{\mathcal{F}}} f_{Y}(\boldsymbol{y}(\mathbf{r}, \theta)) d \boldsymbol{y}
$$

with the joint probability function $f_{Y}(\boldsymbol{y}(\mathbf{r}, \theta))$ of the random-field valued model responses. Since the integral equation is high-dimensional in realistic engineering problems, its solution is usually approximated using Monte Carlo analysis or one of its more advanced variants [29, 30, 31, 32]. 
In case also interval-valued parameters are present in the numerical model, $\boldsymbol{y}(\mathbf{r}, \theta)$ becomes a P-box, and consequently $P_{f}$ becomes interval valued [31]. In this case, the non-determinism in the model responses should be separated in the estimation of the bounds of the failure probability. Following a hyper-cubic approximation of the solution set $\tilde{\boldsymbol{y}}$, the interval valued $P_{f}^{I}$ is expressed as:

$$
\begin{aligned}
P_{f}^{I} & =\left[\underline{P}_{f}, \bar{P}_{f}\right] \\
& =\left[\min _{\tilde{y}} \int_{\mathcal{Y}_{\mathcal{F}}} f_{Y}(\boldsymbol{y}(\mathbf{r}, \theta)) d \boldsymbol{y} ; \quad \max _{\tilde{y}} \int_{\mathcal{Y}_{\mathcal{F}}} f_{Y}(\boldsymbol{y}(\mathbf{r}, \theta)) d \boldsymbol{y}\right]
\end{aligned}
$$

175

As such, a double-loop problem is created, where for each realization of the interval field, a probabilistic failure estimation has to be performed. The outer (interval) loop then iterates until the extrema of the failure probability are obtained, similarly to solving a regular interval FE problem. For general nonmonotonous models, the corresponding computational cost is as such intractable when a naive approach that uses global optimization in conjunction with Monte Carlo simulation is applied. A solution hereto can be found in the application of e.g., Advanced Line Sampling 31 or special hybrid propagation strategies proposed by 33 .

In case strict monotonic models are considered, the outer minimization and maximization problems can be relaxed to a combinatorial search of the $2^{n_{b}}$ vertices of $\boldsymbol{H}^{\boldsymbol{I}}(\mathbf{r})$ to determine the bounds on the probability of failure. The algorithm in this case reduces to:

1. check monotonicity by asserting that $\operatorname{sgn}\left(\frac{\partial \boldsymbol{y}}{\boldsymbol{\alpha}_{\boldsymbol{i}}}\right)=c t e, \forall \alpha_{i} \in\left[\underline{\alpha_{i}} ; \overline{\alpha_{i}}\right]$

2. construct realisations of random $\boldsymbol{H}(\mathbf{r}, \theta)$ and interval fields $\boldsymbol{H}^{\boldsymbol{I}}(\mathbf{r})$

3. propagate $\boldsymbol{H}(\mathbf{r}, \theta)$ for each element in $\alpha_{1}^{I} \times \alpha_{2}^{I} \times \ldots \times \alpha_{n_{b}}^{I}$

4. estimate $P_{f}^{I}$ from the p-box valued set $\boldsymbol{y}(\mathbf{r}, \theta)$ according to eq. 14

In case non-monotonicity is encountered, the general form of the transformation method can be applied to replace step (3) 28]. Note furthermore that 195 computational gain is only established for comparably low-dimensional interval 
fields, as the corresponding computational cost scales $\mathcal{O}\left(2^{n_{b}}\right)$. Alternative approaches to limit the computational cost include e.g., surrogate models such as Kriging [34] or Chaos Expansions [35.

\section{Case study}

The application of advanced non-deterministic methods for the design of reliable and robust components has proven its merit in many engineering applications. However, a typical pitfall in the application of these techniques lies in the accurate and realistic modelling of the constituting parameter nondeterminism. Especially in case intra-uncertain parameters are considered, such quantification is challenging. This section aims at illustrating the application of random and interval field approaches for the modelling of respectively spatially variable and spatially uncertain model quantities, stemming directly from the production process. As such, it is possible to assess the product reliability, based on the production process quantities. Specifically, a functional component that is designed using topology optimisation is considered, as illustrated in figure 1 The geometry is fully constrained in the right-most circular hole, and a uniaxial tensile load of $4 \mathrm{~N}$ is applied to the left-most circular hole in the positive $y$-direction, corresponding to a cantilever beam-like loading.

The corresponding displacement fields are obtained by approximating the solution of the conservation laws by means of the finite element (FE) method. Hereto, the geometry is meshed using 15182 linear plane-stress triangular elements with a thickness of $2 \mathrm{~mm}$, yielding a total of 16274 degrees of freedom. This mesh is constructed fine enough as to allow for an accurate random field discretization, as discussed later on. A close-up view of the mesh is illustrated in figure 2

Figure 3 shows a single realisation of the displacement field in the considered component.

For this case study, the part is produced in Poly-Amide 12 (PA-12), using Laser Sintering (LS). It is assumed that the part is produced using a laser energy 


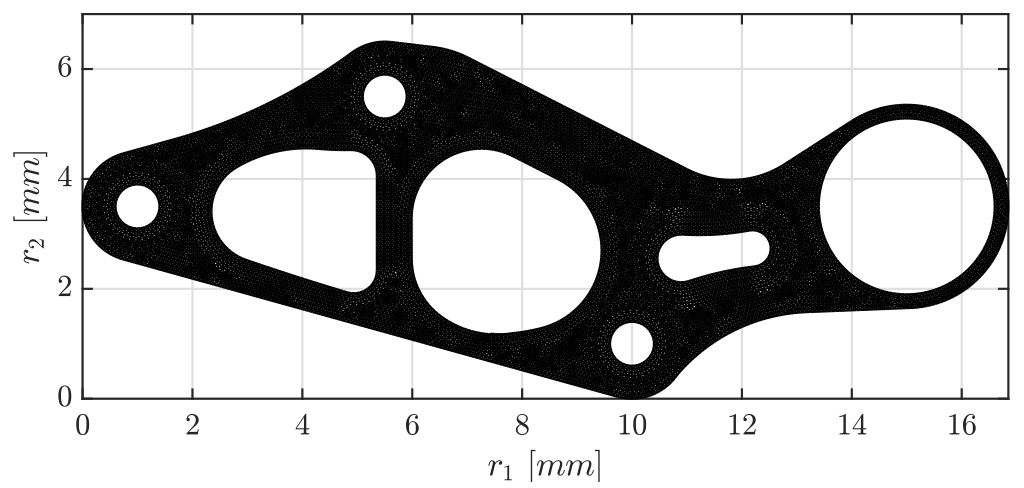

Figure 1: Finite element mesh of the considered case study

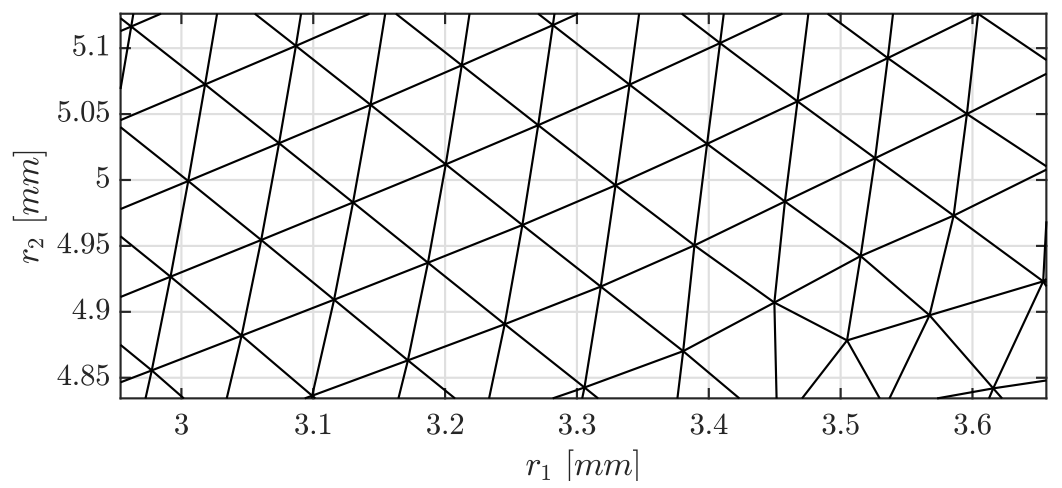

Figure 2: Close-up view of the finite element mesh of the considered case study

density of $35 \mathrm{~mJ} / \mathrm{mm}^{2}$, as this corresponds with the industrial standard. During production, the part is oriented with its $r_{1}$ axis to with the stacking direction of subsequent layers. Since there exist many unquantified sources of variability during the LS process, there exists some uncertainty on important final part quantities [4]. In this case study, both Young's modulus as well as the thickness of the structure are considered to be uncertain as a result of these uncertain process quantities.

To model these sources of non-determinism, a link to the underlying pro- 


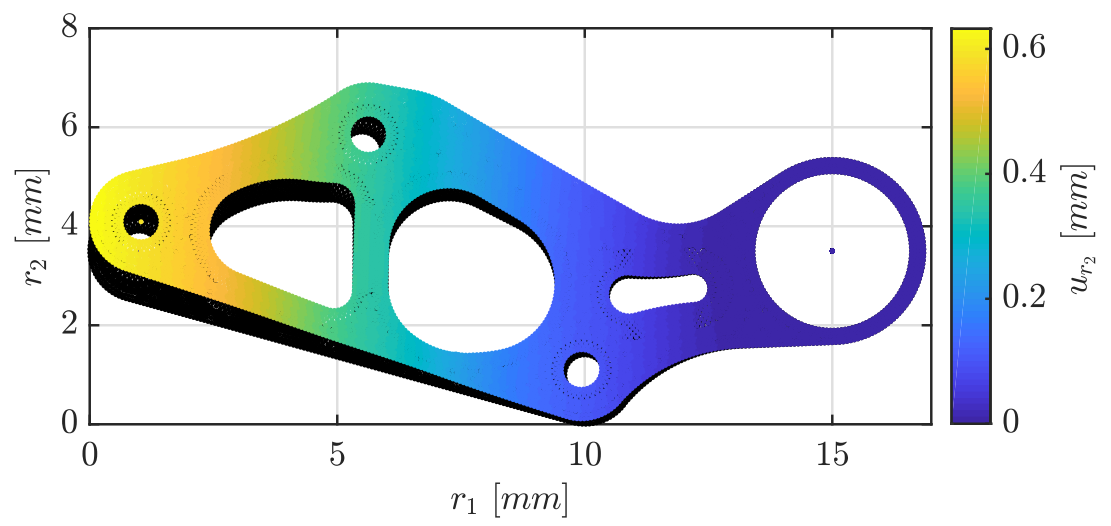

Figure 3: Illustration of the $u_{r_{2}}$ displacement field in the topology optimised component with uncertain Young's modulus and thickness.

duction process has to be established to base the estimated parameter values on first-principle based process knowledge. The influence of many process-related variables on the mechanical and dimensional performance of Laser Sintered PA12 components has been illustrated in literature (see e.g. [8, 1, 36]). Recent work by the authors showed in this context that the quality of laser sintered parts in terms of local porosity content, cross-sectional area and mechanical performance is affected by thermo-temporal effects such post-scan cooling rate and the powder flow time and corresponding inter-particle coalescence. These effects are directly impacted by process variables such as the inter-layer time and laser energy density [4, 6], and pose a challenge for the design of LS-PA12 parts since they are very hard to control during the process. This is a direct result of the fact that these parameters result from the localised heating of the polymer, which has typically a low conductivity, together with the high scan speeds and often complicated geometries of the produced parts.

Since it is very hard to control these effects during production, this case study will illustrate how these effects, quantified on lab-scale test specimens, should be accounted for in a realistic design study. Specifically, it is illustrated how the uncertainty and variability in porosity and local dimensional accuracy can be accounted for. The effect of local porosity is incorporated in the numerical model 
by accounting for local changes in the apparent Young's modulus of the PA-12 material. Since these pores are, given a set of processing parameters, inherently present in Laser Sintered parts, a probabilistic modelling approach is considered to model this inherent variability. As concerns dimensional accuracy, the model is subjected to local uncertainty in thickness. This uncertainty stems directly from other, further undefined, parts that are placed for production in the same build envelope as the considered part, resulting in uncertainty on the inter-layer time. Consequently, since this inter-layer time directly affects the dimensional accuracy, and the root cause for the uncertainty is non-statistical, the local uncertainty is modelled following an interval field approach. It should be noted that the list of considered sources of non-determinism is non-exhaustive, as among others, also effects of the location in the build envelope and/or variability in the flowability of the powder can be considered. However, also for these effects, a similar approach can be followed to include them in the analysis.

\subsection{Local variability}

Porosity in laser sintered parts stems from insufficient consolidation during the production process and is as such a direct result of the interplay between the amount of energy delivered to the material and the time given to the particles to coalesce with neighbouring material. Many parameters affect the local porosity in LS parts, most notably the energy density. Previous research shows that typically, variations in local porosity of $2 \%$ to $8 \%$ on a length scale of $100 \mu \mathrm{m}$ [5, 37] are encountered. These pores both act as stress concentrators 44, and furthermore reduce the load bearing cross-section area [6. As such, they influence the local apparent Young's modulus of the material to a large extent. Under the assumption that the pores are perfectly spherical, the local apparent Young's modulus $E^{*}$ can be computed as:

$$
E^{*}(\mathbf{r})=E(1-\rho(\mathbf{r}))
$$

with $\rho(\mathbf{r}) \in \Omega$ the porosity distribution and $E \in \mathbb{R}$ the global Young's modulus. 
Due to the randomness of the studied phenomenon and its spatial nature, a Gaussian isotropic random field with exponential covariance kernel is con-

realisation of the field over $\Omega$. As may be noted, the spatial structure of the random field realisation, as well as the corresponding values, correspond to the spatial porosity values that are commonly reported in literature. 

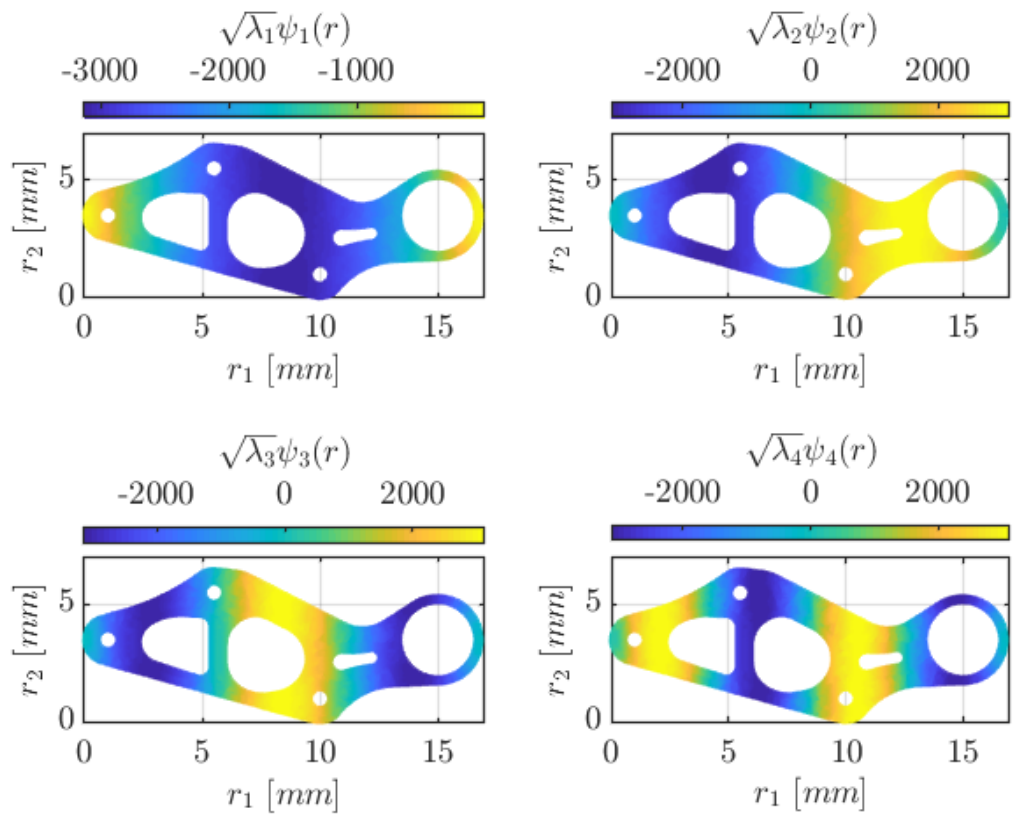

Figure 4: Base function values for one of the realisations of the random field

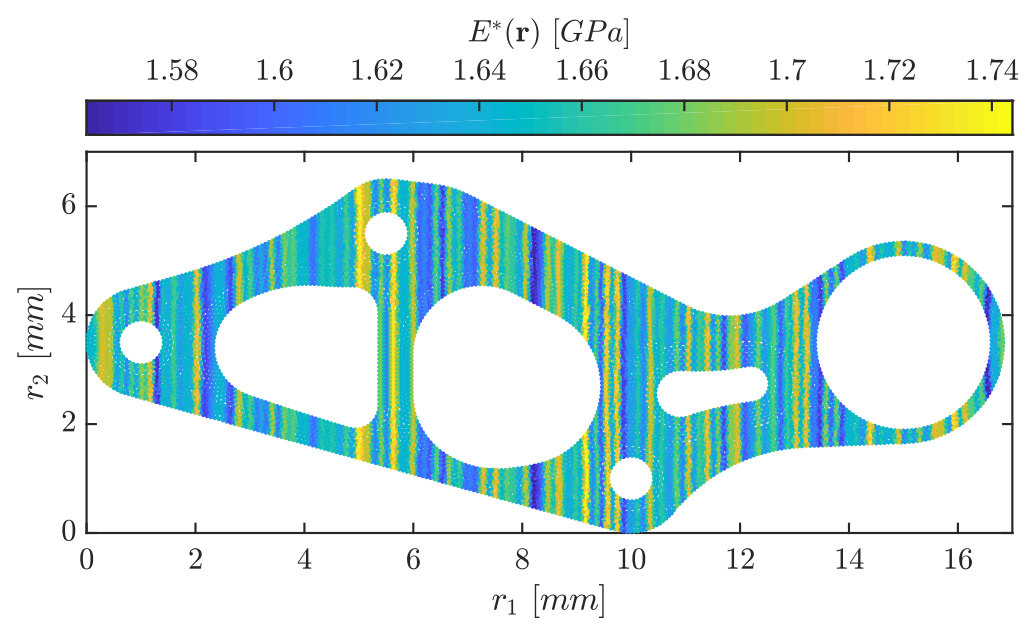

Figure 5: Single realisation of the random field generated over $\Omega$ for Young's modulus of the considered material 


\subsection{Local uncertainty}

300 parts are jointly produced with the rocker is considered. Since the presence of other parts in the build envelope directly affect the inter-layer time, and consequently, the dimensional accuracy of the constructed part [6], this effect should be accounted for in the design, especially since the current trend in LS

Case 2: it is known that the other parts have a prismatic geometry with a cross-sectional area between $95 \mathrm{~mm}^{2}$ and $116 \mathrm{~mm}^{2}$ for the first part, and $50 \mathrm{~mm}^{2}$ and $70 \mathrm{~mm}^{2}$ for the second.

Case 3: it is known that the other parts have a prismatic geometry with a cross-sectional area between $96 \mathrm{~mm}^{2}$ and $99 \mathrm{~mm}^{2}$ for the first part, and $53 \mathrm{~mm}^{2}$ and $65 \mathrm{~mm}^{2}$ for the second.

Case 4: it is known that the other parts have a prismatic geometry with a cross-sectional area of $97.5 \mathrm{~mm}^{2}$ for the first part, and $59 \mathrm{~mm}^{2}$ for the second

325

The corresponding interval fields are constructed by first performing a piecewise linear interpolation to link the area of the other parts $\left(a_{o}\right)$ to the crosssectional area $a_{c}$ data presented by the authors in ([6] - fig. 7) for the $35 \mathrm{~mJ} / \mathrm{mm}^{2}$ 
curve, and interpolating these curves on the $a_{o}$ values listed above as to infer the bounds on the cross-sectional area of the part $a_{c}$. The core underlying assumption is here that the same effect as noted in [6 influences the thickness $t$ of this particular part, and that this effect scales relatively with the size of the part being produced. Evidently, when applied in a real engineering context, such assumption should be validated experimentally on the production machine and material under consideration in a similar approach as [6]. For simplicity reasons, but without losing generality, it is assumed that this effect affects only the thickness $t$ of the part.

A single base function is applied for each "zone" in the part (i.e., the zone without other interfering parts, and the two zones with the unknown parts), and they are constructed following inverse distance weighting interpolation using 340 eq. (9). The control points of these base functions are set such that the zone of influence correlates to the respective zones of influence, and the factor $p$ is heuristically set to 8 as to minimise non-physical mutual influence of the zones on each other by forcing high gradients in the "transition zones" in the base functions. These base functions are illustrated in figure 6, and are constant for all case studies. These base functions are the same for the three case studies since the scale of the uncertainty is only contained in the interval scalars.

Following the explicit interval field formulation, presented in eq. (8), an interval scalar is linked with each base functions. According to the made assumptions, and based on prior work of the authors as discussed above, these interval scalars are taken to be respectively for each case study:

Case1: $\alpha_{1}^{I}=[1.924 ; 1.924] m m, \alpha_{2}^{I}=[1.875 ; 1.882] m m$ and $\alpha_{3}^{I}=[1.886 ; 1.904] \mathrm{mm}$

Case2: $\alpha_{1}^{I}=[1.924 ; 1.924] m m, \alpha_{2}^{I}=[1.876 ; 1.880] \mathrm{mm}$ and $\alpha_{3}^{I}=[1.890 ; 1.900] \mathrm{mm}$

Case3: $\alpha_{1}^{I}=[1.924 ; 1.924] m m, \alpha_{2}^{I}=[1.877 ; 1.879] m m$ and $\alpha_{3}^{I}=[1.892 ; 1.898] \mathrm{mm}$

Case4: $\alpha_{1}^{I}=[1.924 ; 1.924] m m, \alpha_{2}^{I}=[1.878 ; 1.878] m m$ and $\alpha_{3}^{I}=[1.895 ; 1.895] \mathrm{mm}$

355 Note that since case 4 corresponds to exactly known other parts, the thickness in this case is also a deterministic field. 

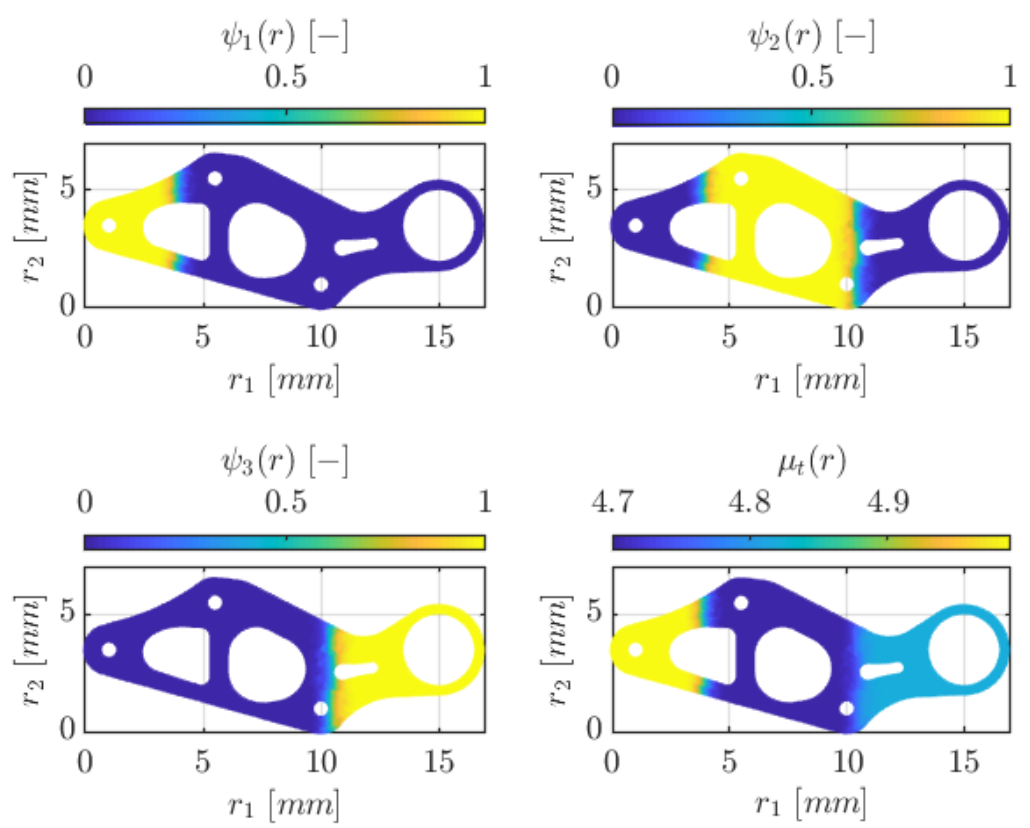

Figure 6: Interval field basis functions $\boldsymbol{\psi}_{i}(\mathbf{r}), i=1, \ldots, 3$, as well as the deterministic field $\mu_{t}(\mathbf{r})$ corresponding to case 4

As such, the spatial nature of the non-determinism is decoupled completely from the amount of uncertainty that is present in the design computation. The extreme realisations of the interval field corresponding to case 1 are illustrated in figure 7

\subsection{Results and discussion}

The combination of interval field uncertainty on the part's thickness and random field uncertainty on Young's modulus is propagated through the numerical model following the double-loop approach discussed in section 2. Since the displacement field in a linearly elastic structure scales monotonously with the part thickness, the reduced transformation method [39] is used for the outer loop (i.e., the propagation of the interval field), which needs in this case $2^{n_{b}=3}=8$ deterministic function evaluations. The inner loop of the problem (i.e., the 

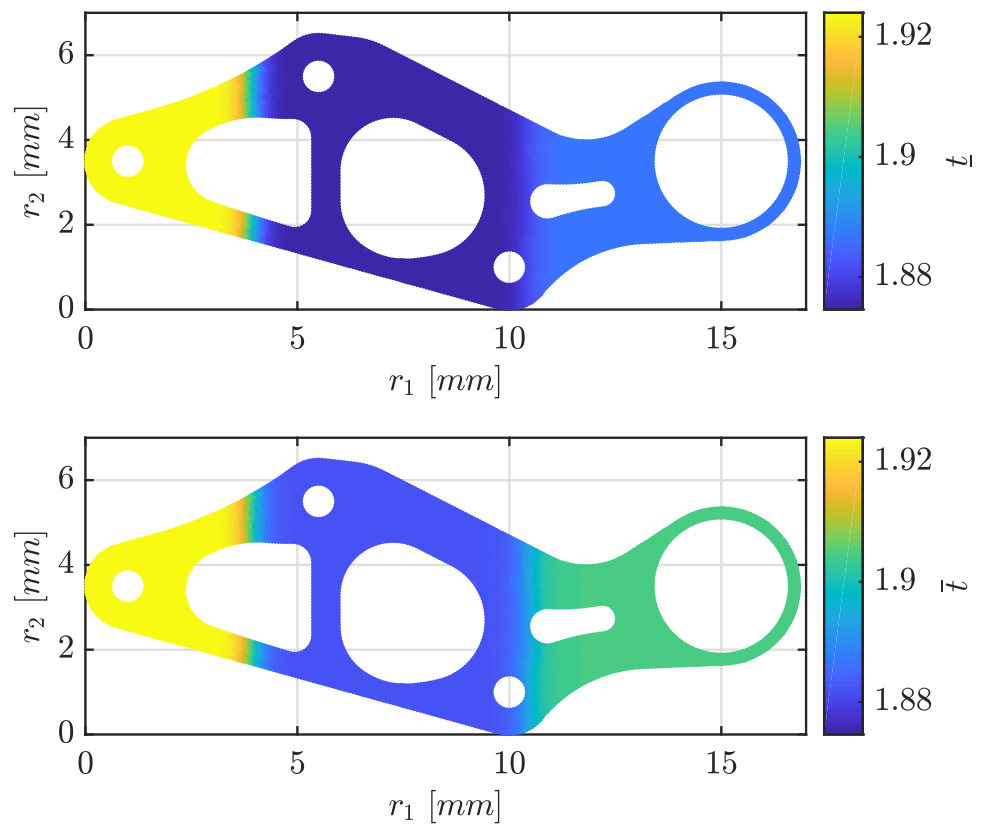

Figure 7: Extremal realizations of the interval field

propagation of the random field for each realisation of the interval field), is con-

ducted by performing a Monte Carlo simulation consisting of 1000 samples of the i.i.d. standard normal variables $\xi_{i}$. As such, a total of 8000 deterministic model evaluations are needed for the propagation of the uncertainty. When performed in parallel on 4 threads of an Intel Xeon E5-1620@3.70 GHz running Xubuntu 16.04 with $32 \mathrm{~Gb}$ RAM, the computation takes $16689 s(\approx 4.6 h)$. The discretization of the 1-dimensional random field takes approx. $120 \mathrm{~s}$, whereas for the interval field this was only approx. $5 \mathrm{~s}$.

Figure 8 illustrates the scatter that is obtained by performing 1000 Monte Carlo simulation for the propagation of the random field on each vertex of the interval field in two nodal displacements in the structure. As may be noted, the non-determinism in these quantities is in case 1 completely dominated by the local uncertainty in Young's modulus. This is a direct result of the comparably large uncertainty that is present on the dimensions of the other parts that 


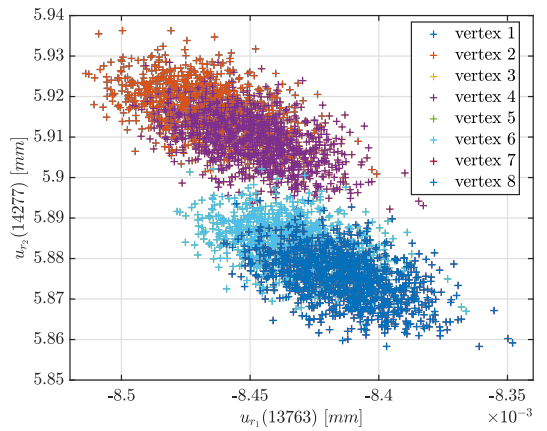

(a) Case 1

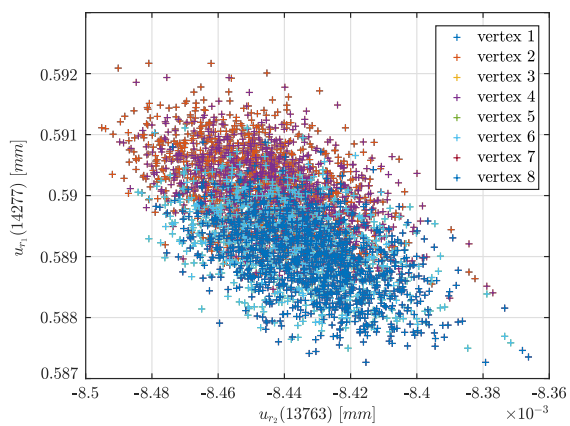

(c) Case 3

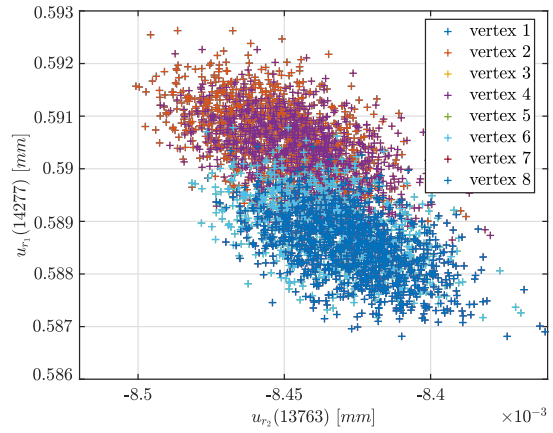

(b) Case 2

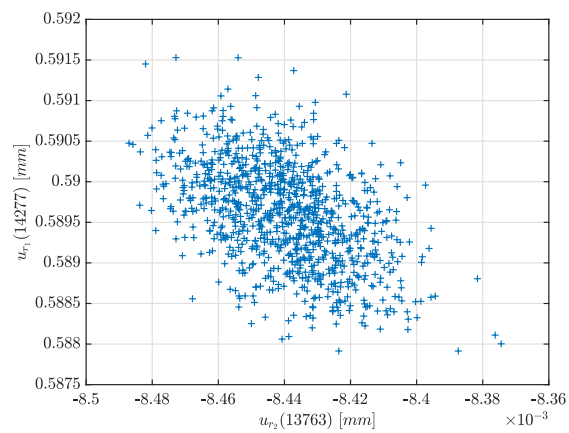

(d) Case 4

Figure 8: Illustration of the scatter in the displacement values in the $r_{2}$ direction for the left-most hole in the structure (node 14277), as well as in the $r_{1}$ direction for node 13763. A point cloud, obtained by Monte Carlo sampling of the random field is shown for each vertex $i$ of the hyper-cubic interval scalars of the interval field.

are in the same build envelope, and hence the inter-layer time. As such, a comparably large uncertainty on the final thickness of the part is obtained. Furthermore, when more information becomes available, the uncertainty in the model's responses is increasingly dominated by the inherent variability that is captured in the random field. In the limit where the other parts are known exactly, the only uncertainty stems from the inherent spatial variability in the local porosity.

Figure 9 shows the empirical distribution functions on the displacement of the left-most hole in the structure. These functions are obtained by performing 
the double loop uncertainty quantification on the three cases of the interval field uncertainty on the part thickness in conjunction with the random field on Young's modulus. As can be noted, when more information on the size of the other parts that are built in the same build envelope is gathered, the predictions on the bounds of the probability of obtaining a certain displacement value, and hence the estimated probability of failure, can be made tighter. Therefore, when designing critical components, care should be taken that the build process planning is made adequately as to ensure that the required design specifications are reached.

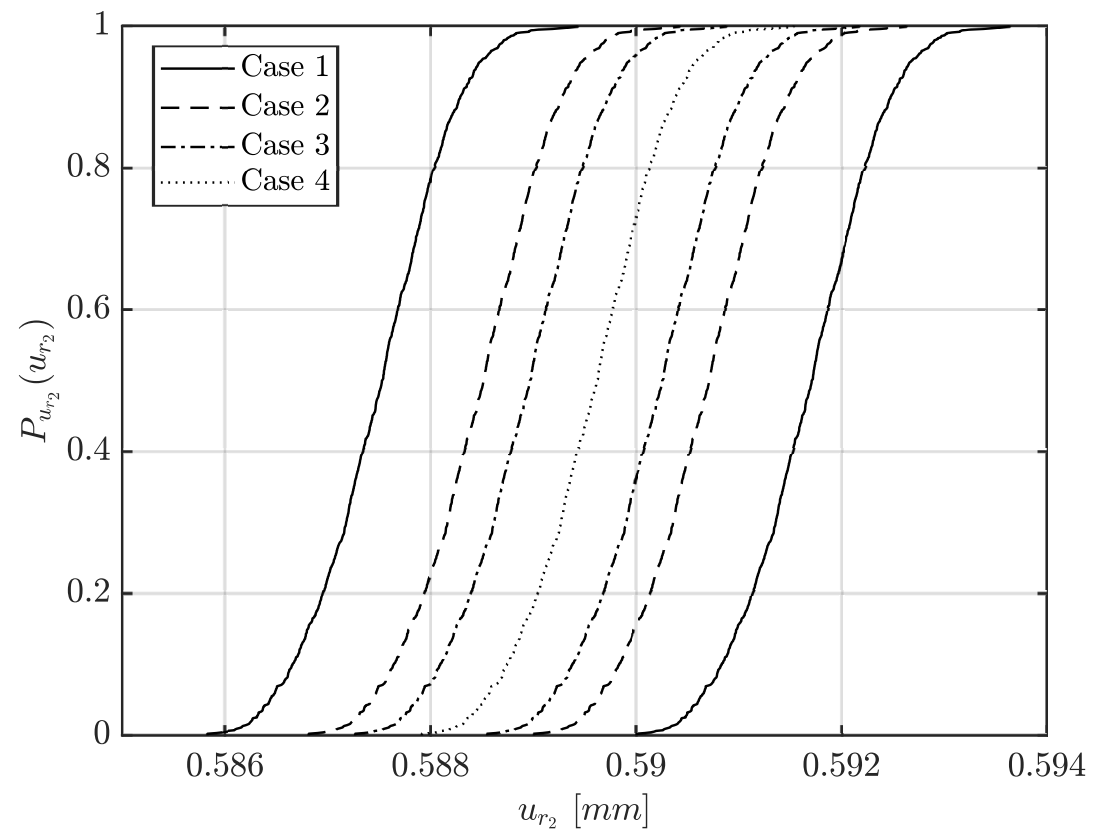

Figure 9: Empirical cumulative distribution functions bounding the hybrid uncertainty resulting from the combination of the random field on Young's modulus with the three cases of interval field uncertainty on the part thickness. Specifically, the displacement of the middle of the left-most hole in the structure is visualised. 


\section{Conclusions}

This paper presents the application of non-deterministic modelling techniques for the propagation of a hybrid set of intra-uncertain and intra-variable model quantities in the design of functional Laser Sintered PA-12 components. A link with the process physics is established to model the non-deterministic quantities. In the context of intra-variability, the spatial porosity distributions that are inherently present in LS PA12 parts are translated to a spatial distribution in the apparent Young's modulus using the random field framework. Furthermore, intra-uncertainty on the final dimensions of other parts that are constructed in the same build envelope as the designed part is linked to uncertainty in the cross-sectional area of the designed part following an interval field approach. As such, a numerical model containing hybrid uncertain fields is obtained, where different sources of non-determinism are taken into account for the design of a functional LS-PA12 component.

The conclusions of the paper can be summarised as follows:

- it is possible to apply highly efficient and established non-deterministic modelling tools to assess the non-determinism in the mechanical behaviour of PA-12 parts produced via Laser Sintering, based on a limited set of experimental data,

- different sources of uncertainty in the design process of Laser Sintered PA-12 components should be separated strictly in the process of model response uncertainty quantification as to prevent unphysical interpretations of these results,

- increasing the knowledge on the production process planning reduces the width of the interval field on the final cross-sectional area of the designed parts, and hence, enables a more crisp prediction of the failure probability, which converges on the uncertainty in the model responses as a result of the inherent variability during the production process. 


\section{Acknowledgements}

430 Foundation (FWO) in the framework of the project "HiDIF: High Dimensional Interval Fields" (project number G0C2218N). Matthias Faes would like to acknowledge the support of the Flemish research foundation for the post-doctoral grant "Generalized inverse Uncertainty Quantification in numerical models" (no. 435

\section{References}

[1] D. L. Bourell, T. J. Watt, D. K. Leigh, B. Fulcher, Performance limitations in polymer laser sintering, Physics Procedia 56 (2014) 147-156.

[2] J. Kruth, X. Wang, T. Laoui, L. Froyen, Lasers and materials in selective 440 laser sintering, Assembly Automation 23 (4) (2003) 357-371. doi:10.1108/ 01445150310698652

[3] D. Moens, D. Vandepitte, Recent advances in non-probabilistic approaches for non-deterministic dynamic finite element analysis, Archives of Com-

[4] M. Faes, Y. Wang, P. Lava, D. Moens, Variability, heterogeneity, and anisotropy in the quasi-static response of laser sintered PA12 components, Strain 53 (2). doi:10.1111/str.12219.

[5] W. Dewulf, M. Pavan, T. Craeghs, J. P. Kruth, Using X-ray computed 450 tomography to improve the porosity level of polyamide-12 laser sintered parts, CIRP Annals - Manufacturing Technology 65 (1) (2016) 205-208. doi:10.1016/j.cirp.2016.04.056

[6] M. Pavan, M. Faes, D. Strobbe, B. V. Hooreweder, T. Craeghs, D. Moens, W. Dewulf, On the influence of inter-layer time and energy density on 
sintering, Polymer Testing 61 (2017) 386 - 395. doi:https://doi.org/ $10.1016 / \mathrm{j} \cdot$ polymertesting. 2017.05 .027

[7] S. Dupin, O. Lame, C. Barrès, J. Y. Charmeau, Microstructural origin of physical and mechanical properties of polyamide 12 processed by laser sintering, European Polymer Journal 48 (9) (2012) 1611-1621. doi:10. 1016/j.eurpolymj.2012.06.007.

[8] R. D. Goodridge, C. J. Tuck, R. J. M. Hague, Laser sintering of polyamides and other polymers, Progress in Materials Science 57 (2) (2012) 229-267. doi:10.1016/j.pmatsci.2011.04.001.

[9] I. Elishakoff, Possible limitations of probabilistic methods in engineering, Applied Mechanics Reviews 53 (2) (2000) 19-36.

[10] F. O. Hoffman, J. S. Hammonds, Propagation of uncertainty in risk assessments: The need to distinguish between uncertainty due to lack of knowledge and uncertainty due to variability, Risk Analysis 14 (5) (1994) 707-712. doi:10.1111/j.1539-6924.1994.tb00281.x.

[11] P. Spanos, R. Ghanem, Stochastic finite element expansion for random media, Journal of engineering mechanics 115 (5) (1989) 1035-1053.

[12] W. Betz, I. Papaioannou, D. Straub, Numerical methods for the discretization of random fields by means of the Karhunen-Loeve expansion, Computer Methods in Applied Mechanics and Engineering 271 (2014) 109-129. doi:10.1016/j.cma.2013.12.010.

[13] R. Ghanem, Ingredients for a general purpose stochastic finite elements implementation, Computer Methods in Applied Mechanics and Engineering 168 (1) (1999) 19-34.

[14] K. K. Phoon, H. W. Huang, S. T. Quek, Simulation of strongly nonGaussian processes using Karhunen-Loeve expansion, Probabilistic Engi口 neering Mechanics 20 (2) (2005) 188-198. doi:10.1016/j.probengmech. 2005.05.007. 
[15] M. Beer, I. A. Kougioumtzoglou, E. Patelli, Emerging Concepts and Approaches for Efficient and Realistic Uncertainty Quantification, in: Maintenance and Safety of Aging Infrastructure, Structures \& Infrastructures Series, CRC Press, 2014, pp. 121-161. doi:doi:10.1201/b17073-6.

[16] R. G. Ghanem, P. D. Spanos, Stochastic Finite Elements: A Spectral Approach, Springer-Verlag New York, Inc., New York, NY, USA, 1991.

[17] W. H. Teichert, Reasons for Uncertainty and their Consequence, in: Proceedings of the 23rd International Conference on Noise and Vibration Engineering, ISMA, Leuven, 1998, pp. 961-966.

[18] D. Moens, M. De Munck, W. Desmet, D. Vandepitte, Numerical dynamic analysis of uncertain mechanical structures based on interval fields, in: IUTAM symposium on the vibration analysis of structures with uncertainties, Springer, 2011, pp. 71-83.

[19] A. Sofi, G. Muscolino, I. Elishakoff, Static response bounds of Timoshenko beams with spatially varying interval uncertainties, Acta Mechanica 226 (11) (2015) 3737-3748. doi:10.1007/s00707-015-1400-9.

[20] W. De Mulder, D. Moens, D. Vandepitte, Modeling uncertainty in the context of finite element models with distance-based interpolation, in: Proceedings of the 1st international symposium on uncertainty quantification and stochastic modeling, 2012.

[21] M. Faes, D. Moens, Identification and quantification of spatial interval uncertainty in numerical models, Computers and Structures 192 (2017) 16-33. doi:10.1016/j.compstruc.2017.07.006.

[22] M. Faes, J. Cerneels, D. Vandepitte, D. Moens, Identification and quantification of multivariate interval uncertainty in finite element models, Computer Methods in Applied Mechanics and Engineering 315 (2017) 896-920. doi:10.1016/j.cma.2016.11.023. 
[23] D. Degrauwe, G. Lombaert, G. D. Roeck, Improving interval analysis in finite element calculations by means of affine arithmetic, Computers \& Structures 88 (3-4) (2010) 247 - 254. doi:http://dx.doi.org/10.1016/ j.compstruc.2009.11.003.

[26] V. Kreinovich, J. Beck, C. Ferregut, A. Sanchez, G. R. Keller, M. Averill, S. A. Starks, Monte-carlo-type techniques for processing interval uncer-

[28] M. Hanss, The transformation method for the simulation and analysis of systems with uncertain parameters, Fuzzy Sets and Systems 130 (3) (2002) 277-289. doi:10.1016/S0165-0114(02)00045-3.

[29] G. I. Schuëller, H. J. Pradlwarter, P. S. Koutsourelakis, A critical appraisal 535

口 neering Mechanics 19 (4) (2004) 463-474. doi:10.1016/j.probengmech. 2004.05 .004 
[30] G. Schuëller, On the treatment of uncertainties in structural mechanics and analysis, Computers \& Structures 85 (5) (2007) 235-243.

[32] M. Faes, M. Broggi, M. Beer, D. Moens, Failure probability under uncertain surrogate model predictions, in: Proceedings of the joint ICVRAM ISUMA

[33] D. Wu, W. Gao, F. Tin-Loi, Y. L. Pi, Probabilistic interval limit analysis for structures with hybrid uncertainty, Engineering Structures 114 (2016) 195-208. doi:10.1016/j.engstruct.2016.02.015.

[34] M. Faes, M. Broggi, M. Beer, D. Moens, Failure probability under uncertain

[31] M. de Angelis, E. Patelli, M. Beer, Advanced Line Sampling for efficient robust reliability analysis, Structural Safety 52 (PB) (2015) 170-182. doi: $10.1016 / j$. strusafe.2014.10.002 UNCERTAINTIES conference, no. 2, Florianopolis, 2018. surrogate model predictions, Proceedings of the joint ICVRAM ISUMA UNCERTAINTIES conference (2).

[35] B. Sudret, Global sensitivity analysis using polynomial chaos expansions, Reliability Engineering \& System Safety 93 (7) (2008) 964-979.

[36] A. Wegner, G. Witt, Correlation of Process Parameters and Part Properties in Laser Sintering using Response Surface Modeling, Physics Procedia 39 (2012) 480-490. doi:10.1016/j . phpro.2012.10.064.

[37] M. Pavan, T. Craeghs, J.-P. Kruth, W. Dewulf, Understanding the link between process parameters, microstructure and mechanical properties of laser sintered pa12 parts through x-ray computed tomography, Proceedings of the 2nd International Conference on Progress in Additive Manufacturing (Pro-AM 2016) (2016) 569-574.

[38] W. Dewulf, M. Pavan, T. Craeghs, J.-p. Kruth, Using X-ray computed tomography to improve the porosity level of polyamide-12 laser sintered parts, CIRP Annals - Manufacturing Technology 65 (1) (2016) 205-208. doi:10.1016/j.cirp.2016.04.056 
[39] M. Hanss, Applied Fuzzy Arithmetic: an Introduction with Engineering Applications, Springer, Berlin, 2005. 